\title{
Antimicrobial resistance and in vitro biofilm-forming ability of Enterococci spp. isolated from urinary tract infection in a tertiary care hospital in Dhaka
}

\author{
Akhter $\mathrm{J}^{1}$, Ahmed $\mathrm{S}^{2}$, Saleh $\mathrm{AA}^{2}$, Anwar $\mathrm{S}^{2}$ \\ ${ }^{1}$ Chest Disease Clinic, Chittagong, ${ }^{2}$ Department of Microbiology \& Immunology, \\ Bangabandhu Sheikh Mujib Medical University, Shahbag, Dhaka. Email: jabinakhter@yahoo.com
}

\begin{abstract}
The biofilm mode of life conveys a survival advantage to the microorganism associated with it. Biofilm on an indwelling urinary catheter consists of adherent microorganisms, their extra cellular products, and host components deposited on the catheter and thus biofilm on urinary catheters results in persistent infections that are resistant to antimicrobial therapy. This study was done during the period of January 2010 to December 2010. Fifty nine enterococci isolated from 1203 urine samples were speciated by conventional microbiological methods and examined for their ability to form biofilm by microtitre plate assay and antimicrobial susceptibility testing by disc diffusion method for 10 clinically relevant antibiotics respectively. Biofilm producing Enterococci were more frequently found in catheterized than in non catheterized patient ( $\mathrm{p}<0.004)$. Enterococcus faecium showed increased resistantance to multiple antibiotic than Enterococcus faecalis. Significant relationship was found between biofilm production with antibiotic resistance to amoxicillin, co-trimoxazole, ciprofloxacin, gentamycin, cefotaxime, and cefuroxime. This study demonstrated a high propensity among the isolates of Enterococci to form biofilm and a significant association of biofilms with multiple drug resistance.
\end{abstract}

\section{Introduction}

Enterococci, recognized as opportunistic pathogens, are natural inhabitants of the oral cavity, normal intestinal microflora, and female genital tract of both human and animals. They are the important global cause of nosocomial infections, being increasingly associated with urinary tract infections, endocarditis, intra-abdominal and pelvic infections, catheter-related infections, surgical wound infections, and central nervous system infections ${ }^{1}$. It ranked second after Pseudomonas aeruginosa among the nosocomial pathogens. Furthermore it is emerging recently as a major cause of hospital acquired infections because of the extent of its antimicrobial resistance and its propensity to cause large, often multi-facility nosocomial outbreaks. Infections due to Enterococci often prove difficult to treat due to high level of resistance to multiple antibiotics as a result of both intrinsic and acquired mechanisms. The potential ability of Enterococci to form biofilms might also explain its outstanding antibiotic resistance, survival properties and increased virulence.

The two most common Enterococci species are Enterococcus faecalis and Enterococcus faecium. Both are capable of producing biofilms, which consist of a population of cells attached irreversibly on various biotic and abiotic surfaces, encased in a hydrated matrix of exopolymeric substances ${ }^{2}$. Biofilms are notoriously difficult to eradicate and are a source of many chronic infections. Bacteria in biofilms colonize a wide variety of medical devices, such as catheters, artificial cardiac pacemakers, prosthetic heart valves and orthopaedic appliances ${ }^{2}$. A mature biofilm can tolerate antibiotics at concentrations of 10-1000 times more than are required to kill planktonic bacteria. Bacteria in biofilms are resistant to phagocytosis, making biofilms extremely difficult to eradicate from living hosts ${ }^{3}$. Enterococci in biofilms are more highly resistant to antibiotics than planktonically growing Enterococci, thus the potential impact of biofilm formation could be significant. Therefore, the present study was undertaken on clinical isolates of Enterococci causing UTI to determine the frequency of biofilm formation by microtitre plate method and correlate biofilm formation with multiple antibiotic resistances.

\section{Materials and Methods}

In this study the Enterococci were isolated from urine of suspected urinary tract infection patients of different age and sexes from outpatient and inpatient department of BSMMU, Dhaka. This 
study was done during the period of January 2010 to December 2010. A total of 1203 urine samples were tested for the isolation of Enterococci. All samples were initially cultured on chromogenic agar media (Difco laboratories, Detroit, USA) [Figure 1]. They were sub cultured on blood agar plates at $37^{\circ} \mathrm{c}$ for $24 \mathrm{hrs}$. All suspected colonies were identified by standered microbiological methods including gram staining, catalase test and esculin hydrolysis test, growth in $6.5 \% \mathrm{NaCI}$ and at $\mathrm{pH} 9.6^{4}$.

Enterococci isolates were identified to the species level by using conventional physiological tests devised by Facklam and Collins ${ }^{5}$ which are based on carbohydrate fermentation using $1 \%$ solution of following sugars: mannitol, sorbitol, arabinose raffinose, sorbose and pyruvate utilization in $1 \%$ pyruvate broth; arginine decarboxylation in Moellers decarboxylase broth; motility was determined in modified Difco motility medium, and pigmentation was observed after overnight growth on tryptic soy agar ${ }^{4}$.

Antibiotic sensitivity testing: Antibacterial resistance pattern of 10 antimicrobial agents were performed with Amoxicillin, Cotrimoxazole, Ciprofloxacin, Nitrofurantoin, Ceftriaxone, Gentamycin, Cefotaxime, Cefuroxime, Imipenem, Vancomycin using standard disk diffusion method (Kirby- Bauer sensitivity test) ${ }^{6}$ susceptibility test. All test were performed on Muller Hinton agar (Oxoid Co, Hampshire, UK), and results read after $24 \mathrm{hrs}$ of incubation at $37^{\circ} \mathrm{c}$.

Biofilm Assay: The ability of the Enterococci isolates to form biofilm on an abiotic surface was described by Toledo-Arena $e t a l^{7}$. Bacterial strains to be tested were grown in BHI broth containing $0.25 \%$ glucose and incubated overnight. Overnight broth cultures were diluted 1:20 in fresh BHI broth supplemented with glucose. Two hundred $\mu 1$ of diluted strain was dispensed into triplicate wells in a single column of a sterile 96-well flat-bottom microtiter plate (Becton Dickinson) \& incubated at $37^{\circ} \mathrm{C}$ for 24 hours. Planktonic cells were discarded by tapping the microtitre plate, and the wells were washed three times with sterile phosphate-buffered saline (PBS).The plates were inverted and allowed to dry for $1 \mathrm{~h}$ at room temperature. Two hundred $\mu \mathrm{l}$ of $0.5 \%$ aqueous crystal violet solution was added to each well \& allowed to stand for 15 minutes. The wells were subsequently washed thrice with sterile PBS to wash off the excess crystal violet. Two hundred $\mu 1$ of $80: 20(\mathrm{vol} / \mathrm{vol})$ mixtures of ethyl alcohol and acetone was added to solubilized bound crystal violet. Absorbance of the extracted crystal violet was measured at $550 \mathrm{~nm}$ automatic microplate reader (ELX-800 Bio-Tek Instruments,
Inc., Winooski, Vt.). For positive control Staphylococcus epidermidis and for negative control non-biofilm forming bacteria salmonella typhi was used in each plate. All biofilm assays were repeated three times. The cut-off value (ODc) was established. ODc, defined as three standard deviations (SD) above the mean OD of the negative control. Final OD value of a tested strain was expressed as average OD value of the strain reduced by ODc value of the triplicate assays. Any OD value above the cutoff value was indicative of biofilm production ${ }^{8}$. In the quantitative assay for the biofilm production, the isolates were classified as strongly biofilm producing (strongly adherent), weakly adherent isolates and non-biofilm producers (non-adherent).

\section{Results}

Fifty-nine enterococci were isolated from total a total of 1203 urine samples collected from patients with suspected urinary tract infection were studied during the study period corresponding to $8.44 \%$ of all positive urine cultures. Of them $13.84 \%$ Enterococci were isolated from patient on indwelling urethral catheter. Based on the biochemical reactions, only 55 enterococci could be speciated. E. faecalis (71.18\%) was the most common species identified followed by E. faecium $10(16.94 \%)$.

Quantitative microtitre assay for biofilm formation was strongly positive in 38 isolates $(64.40 \%)$ while the remaining isolates were either weakly adherent (21) or non-biofilm producers considered as negative or non-biofilm producers. Biofilm positive enterococci were more frequently found in catheterized patient than that of mid stream urine. Isolates screened for biofilm formation by microtitre plate assay method are shown in [Fig.2].

Among the isolates tested, 6(14.28\%) E. faecalis of $42 \& 2(20 \%)$ E. faecium of 10 was resistant to nitrofurantoin. Increased resistance was noted in E. faecium to amoxicillin $(90 \%)$, cotrimoxazole (80\%) ciprofloxacin (70\%), ceftriaxone (90\%), gentamycin $(80 \%)$ cefotaxime $(90 \%)$ and cefuroxime $(80 \%)$. Isolates of E. faecalis were resistant to amoxicillin $(66.66 \%)$, cotrimoxazole $(71.42 \%)$ ciprofloxacin $(76.19 \%)$, ceftriaxone $(64.28 \%)$, gentamycin $(71.42 \%)$ cefotaxime $(85.71 \%)$ and cefuroxime $(80.95 \%)$. Almost all the multiple antibiotic resistant isolates tested positive for biofilm formation. Regarding the antimicrobial sensitivity pattern, the Enterococcal isolates were best sensitive to vancomycin $(98.30 \%)$, followed by imipenem $(94.91 \%)$ and nitrofurantoin $(86.44 \%)$. Decreased sensitivity to amoxicillin, ciprofloxacin, ceftriaxone, cefotaxime and 
cefuroxime were observed in Enterococci isolated ranging from $23 \%$ to $38 \%$.

Statistical analysis showed significant relationship of biofilm formation with antibiotic resistance. Biofilm formation were significantly found in $30(81.08 \%)$ of 37 amoxicillin resistant isolates, in $32(84.21 \%)$ of 38 cotrimoxazole resistant enterococci, in $32(80 \%)$ of 40 ciprofloxacin resistant isolates, in $34(89.47 \%)$ of 38 gentamycin resistant isolates and in 30(75\%) of 40 cefotaxime resistant Enterococci (Table I) [Fig. 3].

Table 1: Association between virulence factors Biofilm production and antibiotic susceptibility pattern of Enterococci isolated from urinary tract infection $(\mathrm{N}=59)$.

\begin{tabular}{lccc}
\hline Antimicrobials & $\begin{array}{c}\text { No. of resistant } \\
\text { isolates producing } \\
\text { biofilm/Total no. } \\
\text { of resistant isolates }\end{array}$ & $\begin{array}{c}\text { No.of sensitive } \\
\text { Isolates producing } \\
\text { biofilm/Total no. } \\
\text { of sensitive isolates }\end{array}$ & $\begin{array}{c}\mathrm{P} \\
\text { value }\end{array}$ \\
\hline Amoxicillin & $30 / 37(81.08)$ & $08 / 22(36.36)$ & 0.001 \\
Cotrimoxazol & $32 / 38(84.21)$ & $06 / 21(28.57)$ & 0.001 \\
Ciprofloxacin & $32 / 40(80)$ & $06 / 19(31.57)$ & 0.001 \\
Nitrofurantoin & $08 / 08(100)$ & $30 / 51(58.82)$ & 0.001 \\
Ceftriaxone & $28 / 36(77.77)$ & $09 / 23(39.13)$ & 0.002 \\
Gentamycin & $34 / 38(89.47)$ & $4 / 11(36.36)$ & 0.001 \\
Cefotaxime & $30 / 40(75)$ & $08 / 19(42.10)$ & 0.013 \\
Cefuroxime & $30 / 42(71.42)$ & $08 / 17(47.05)$ & 0.013 \\
Imepenem & $2 / 3(66.66)$ & $36 / 56(64.28)$ & 0.933 \\
Vancomycin & $1 / 1(100)$ & $37 / 58(63.79)$ & 0.453 \\
\hline
\end{tabular}

Figures within the parentheses indicate percentage.

$P<0.001$ Highly significant $P<0.01$ Moderately significant

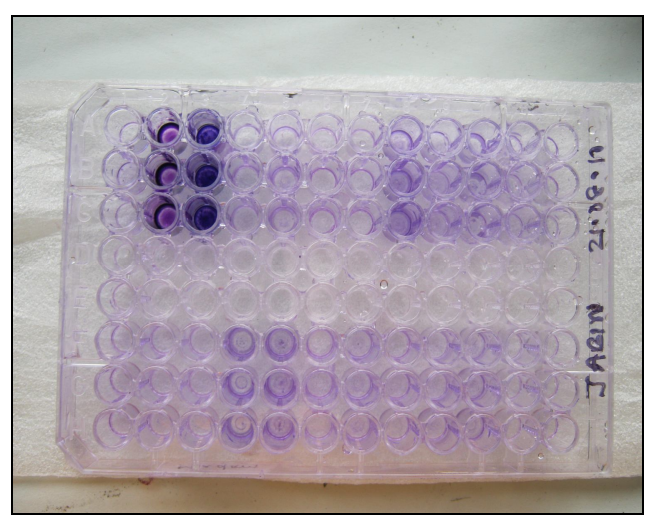

Fig. 1: Biofilm formation by Enterococci

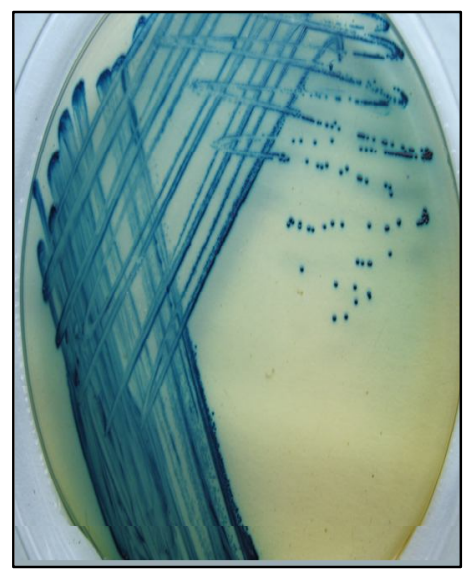

Fig.2: Enterococci in chromogenic agar media

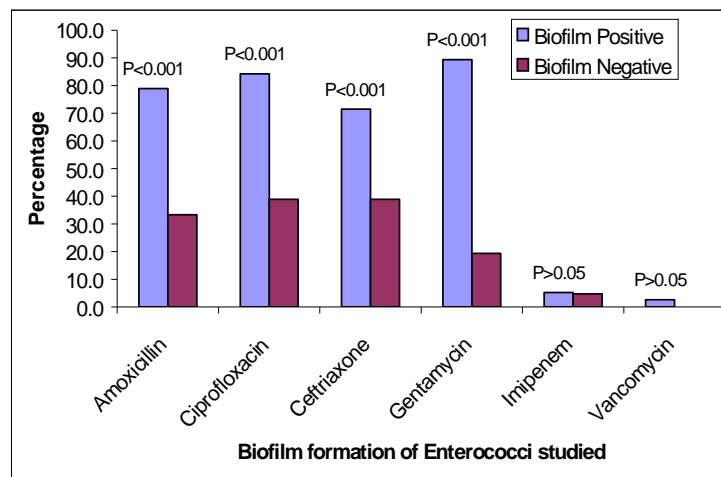

Fig. 3: Comparison of biofilm positive \& biofilm negative isolates among antibiotic resistant Enterococci. The y-axis represents the percentage of the isolates resistant to different antibiotics \& the xaxis represents resistance to the antibiotic mentioned.

\section{Discussion}

Urinary tract infection is one of the most common infectious conditions in clinical practice. ${ }^{9}$. UTIs are mainly caused by Gram-negative microorganisms of the intestinal flora, Enterococcus species, although less common \& despite their lack of pathogenicity, can cause UTI both within and outside hospital settings ${ }^{\mathbf{1 0}}$ and emerged as a major cause of nosocomial infections with alarming rates of multi-drug resistance ${ }^{11}$. In this study majority of the isolates were E. faecalis $42(71.18 \%)$ found both in catheterized \& non-catheterized patients, while E.faecium accounted for $10(16.94 \%) \&$ was more frequent in catheterized urine.

Importance of biofilm formation has been described in the control of microbial infection in several areas because the biofilm can increase resistance to various physical and chemical agents, especially antibiotics 1 . Because of marked differences in biofilm formation and infection type caused by the two predominant species, comparisons between the antibiotic resistances with the formation of biofilm were carried out separately for each species in this study. In our study we found that the majority of biofilm producing bacteria was form catheterised patients $(83.33 \%)$ and $56.09 \%$ from non-catheterized patients. Among them $71.42 \%$ E.faecalis \& $50 \%$ E.faecium produced biofilm. Biofilm positive Enterococci were more frequently found in catheteter urine than in non-catheter urine $(\mathrm{P}<0.05)$. Similarly, Donlan $^{12,13}$ reported in his study the association of biofilm producing bacteria with urinary catheter. Rakhshanda et al $^{14}$ stated in a study that biofilm production by uropathogen was detected in $75 \%$ of the isolates from UTI. Biofilm were mainly detected from Staphylococcus aureus $75 \%$, E.faecalis 75\%, \& Esch.coli 40\%. Enterococci are the one of the causative organism of UTI \& biofilm 
formation allows the strains to persist for a long time in the genitourinary tract and interfere with bacterial eradication. It is hypothesized that biofilm has a function analogous to fimbria of Esch.coli \& Proteus in adherence to the uro-epithelium ${ }^{\mathbf{1 5}}$. Survival advantages conferred by the biofilm community include resistance to phagocytosis, and to antimicrobial agents. Biofilm formation is indirect evidence of adhesiveness \& microtitre plate assay is the indirect way to measure adhesion of Enterococci.

Biofilm exhibits more resistance to broad spectrum antibiotics $^{16}$. This supports that biofilm adds to the virulence profile of microorganism ${ }^{17}$. Resistance to several antimicrobial agents was prevalent among the Enterococci isolates recovered in the hospital. This study investigated the species occurrence and antibacterial resistance pattern of enterococci isolated from UTI individuals. E.faecium was found to be more multidrug resistant than E.faecalis. E.faecium isolates were highly resistant to gentamycin (80\%), amoxicillin (90\%), ciprofloxacin $(80 \%)$, and ceftriaxone $(90 \%)$. But regarding the antimicrobial sensitivity pattern, Enterococcal isolates were best sensitive to vancomycin $(98.30 \%)$, followed by imipenem $(94.91 \%)$ and nitrofurantoin $(86.44 \%)$. Significant relationship was found between biofilm production with antibiotic resistance to amoxicillin, cotrimoxazole, ciprofloxacin, gentamycin, cefotaxime and cefuroxime. Biofilm formation were significantly found in $81.08 \%$ of 37 amoxicillin resistant isolates, in $84.21 \%$ of 38 cotrimoxazole resistant enterococci, in $80 \%$ of 40 ciprofloxacin resistant isolates, in $55.26 \%$ of 38 gentamycin resistant isolates and in $75 \%$ of 40 cefotaxime resistant Enterococci.

Conclusion: According to the findings of this study, we conclude that biofilm forming microorganisms show resistance to multiple antibiotics and nitrofurantoin can be considered a good alternative therapy in enterococcal UTIs because of the lower resistance.

\section{References}

1. Murray, B. E. \& Weinstock, G. M. Enterococci: new aspects of an old organism. Proc Assoc Am Physicians 1999; 111: 328-34.

2. Costerton, J. W., Stewart, P. S. \& Greenberg, E. P. Bacterial biofilms: a common cause of persistent infections. Science 1999; 284: 1318-22.

3. Lewis, K. Riddle of biofilm resistance. Antimicrob Agents Chemother 2001; 45: 999-1007.
4. Fackland R, Sham DA, Teixeira LM: Enterococcus. In Manual of Clinical Microbiology 7th edition. Edited by: Murray PR, Baron EJ, Pfaller MA, Tenover FC, and Yolken RH. Washington: AmericanSociety of Microbiology 1999; 297-05.

5. Facklam RR and Collins MD. Identification of Enterococcus Species Isolated from Human Infections by a conventional test scheme. $J$ of Clin Microbiol 1989; 27:731-4.

6. Bauer AW, Kirby WM, Sherris JC,Turck M. Antibiotic susceptibility testing by a standardized single disk method. Am J Clin Pathol 1966; 45(4):493-6.

7 Toledo-Arena A,Valle J, Solano C, Arrizubieta MJ, Cucarella C, Lamata M, et al., The Enterococcal surface protein, Esp, is involved in Enterococcus faecalis biofilm formation. Appl and Environmental Microbiol 2001; 67: 4538.

8. Stepanovic S, Vukovic D, HolaV, Bonaventura GD, Djukic S, Irkovic IC, Ruzicka F. Quantification of biofilm in microtiter plates: overview of testing conditions and practical recommendations for assessment of biofilm production by staphylococci. APMIS 2007; 115: 891-9.

9. Kunin CM. Care of the urinary catheter. In: Urinary tract infections: detection, prevention and management. Fifth ed. Baltimore: Williams and Wilkins 1997; p. 227-99.

10. Courvalin P. Vancomycin resistance in Gram-positive cocci. Clin Infect Dis 2006; 42 Suppl 1: 25-4.

11. Robert C, Moellering Jr. Principle of anti-infective therapy: Enterococcus species, Streptococcus bovis, and Leuconostoc species. In: Mandell G., Bennett J.E., Dolin R., eds. Principles and practice of infectious disease. 6th ed. Philadelphia: Churchill Livingstone 2005; 1826-5.

12. Donlan RM. Biofilms and device associated infections. Emerg infect Dis 2001; 7:277-81.

13. Donlan RM, Costerton W. Biofilms:survival mechanisms of clinically relevant Microorganisms. Clin Microbiol Rev 2002; 15(2): 167-93.

14. Rakhshanda B, Mubashir A, Ghulam R. Urinary tract infection in diabetic patients and Biofilm formation of Uropathogens. Infectious Diseases Journal of Pakistan 2008; 17(1):7-9.

15. Vergis N, Shankar N, Chow J. et al. (2002) Association between the pre-sence of enterococcal virulence factors gelatinase, hemolysin, and enterococcal surface protein and mortality among patients with bacteremia due to Enterococcus faecalis. CID 2002;35.

16. Mathur T, Singhal S, Khan S, Uphadtay Fatma, T Rattam. A Detection of Biofilm formation among clinical isolates of Staphylococci: An evaluation of three different screening methods. Indian $\mathrm{J}$ Med. Microbial 2006; 24:25-9.

17. Suman E, Jose J, Varghese S, Kotian MS. Study of biofilm production in Escherichia coli causing urinary tract infection. India J Med Microbiol 2007; 25: 305-6. 